\title{
HAití: TRIBUlACIONES DE UN ESTADO COLAPSADO
}

\author{
Haiti: Tribulations of a Failed State
}

\author{
ANDREAS FELDMANN \\ Instituto de Ciencia Política, Pontificia Universidad Católica de Chile
}

\section{JUAN ESTEBAN MONTES}

Programa de Chile, Universidad de Notre Dame

\begin{abstract}
RESUMEN
Este ensayo revisa los principales acontecimientos políticos ocurridos en Haití durante los últimos 22 meses. Durante este período Haití ha logrado cierto grado de estabilización política gracias a la realización de comicios y la posterior elección de René Preval como Presidente. El país ha logrado algunos avances en materia de seguridad y ha estabilizado su economía, la que creció moderadamente. Los avances han sido posibles, en parte, gracias a la presencia de tropas de la Misión de Estabilización de las Naciones Unidas en Haití (MINUSTAH) que han colaborado con las autoridades en diversas áreas claves, sobre todo en materia de seguridad. Más allá de los aspectos positivos, la situación general de Haití sigue siendo extremadamente crítica dado los graves problemas estructurales que enfrenta el país y a su apreciable dependencia de la comunidad internacional.
\end{abstract}

Palabras clave: Sistema Político, MINUSTAH, Seguridad Interna, Estado de Derecho, Derechos Humanos.

\begin{abstract}
This article reviews the main political developments in Haiti in the last 22 months. During this period, the country has attained some degree of political stability as a result of the successful completion of an electoral process and the concomitant election of René Preval as President of the country. Haiti, furthermore, has seen some improvements in security and economic stability triggered by a moderate economic growth. These achievements have been partly possible due to the presence of the United Nations Stabilization Mission in Haiti (MINUSTAH), which, jointly with the Haitian State, have worked to tackle acute problems, in particular lack of security. These improvements notwithstanding, the general outlook of the country and its political stability remain fragile given its significant structural problems and its extensive dependence from the international community.
\end{abstract}

Key words: Political System, MINUSTAH, Internal Security, Rule of Law, Human Rights.

\section{INTRODUCCIÓN}

El 2007 fue probablemente uno de los años más positivos para Haití de la última década. El país gozó de crecimiento económico y de relativa seguridad, algo que no se había producido en varios años. Tras 22 meses de relativa calma, sin embargo, en el mes de octubre se abrieron grietas en la coalición de gobierno, algo que amenaza con desatar 
una nueva crisis política. Los problemas surgen de la decisión de posponer la elección de un tercio de la cámara alta, acusaciones de corrupción en contra de varios miembros del Poder Legislativo y, principalmente, de la decisión del actual presidente, René Préval, de promover cambios a la Constitución del país. A comienzos de 2008 se agudizó una crisis de desabastecimiento alimenticio que ha generado masivas protestas en el mes de abril, a esto se ha acompañado un recrudecimiento de la violencia y los secuestros con fines económicos. Dado este contexto, la situación general de Haití sigue siendo extremadamente crítica, lo que implica que no se puede ser muy sanguíneo con respecto al futuro de esta nación caribeña de 8,5 millones de habitantes. La tesis de este artículo es que a pesar de algunos desarrollos positivos, el futuro de la primera República independiente de América Latina es incierto, en particular porque sus grados de dependencia de la comunidad internacional son excesivamente altos.

El siguiente ensayo pasará revista a los principales acontecimientos ocurridos en Haití durante los últimos 22 meses, hasta abril de 2008. El trabajo está dividido en tres secciones. La primera reseñará brevemente el contexto político y de seguridad del país de la última década. En segundo término, se examinará el desempeño de la nueva administración encabezada por el presidente René Préval, quien asumió el mando del país en mayo del 2006. Esta sección analizará diversos aspectos, entre ellos, la situación de seguridad del país, el desempeño de la economía, las relaciones con el Poder Legislativo y la actuación del Poder Judicial. La tercera sección examinará el rol de la Misión de Estabilización de las Naciones Unidas en Haití (MINUSTAH) y, de manera más general, el papel jugado por la comunidad internacional en la pacificación del país. Finalizamos el ensayo con algunas observaciones y conjeturas sobre el futuro del país en el corto plazo.

\section{EL CONTEXTO DE UN REPUNTE INESPERADO PERO MUY BIENVENIDO}

Haití ha padecido de una aguda crisis sociopolítica durante la mayor parte del siglo XX. Esta crisis ha continuado en lo que va del nuevo siglo. Los problemas derivan principalmente de la inexistencia de un Estado capaz de llevar a cabo funciones mínimas (i.e., prodigar seguridad a sus habitantes, promover el desarrollo económico, proveer una infraestructura mínima, garantizar y promover los derechos humanos) y en donde la inmensa mayoría de los habitantes hacen de la privación un modo de vida. En Haití decenas de miles de personas no cuentan con las condiciones mínimas para asegurar su subsistencia, pues no tienen trabajo ni tampoco acceso a servicios sociales básicos como vivienda, salud, educación, acceso a agua potable o transporte público, entre otros.

Parte del problema es que durante muchos años el Estado -como durante la dinastía política de los Duvallier, que gobernó el país por casi treinta años- se constituyó en un instrumento de los gobernantes para enriquecerse y mantener sus privilegios y prerrogativas. Algunos estiman que Haití padeció regímenes sultanísticos caracterizados por nepotismo, corrupción, venalidad, autoritarismo y represión a gran escala (Chehabi y Linz 1998: 7; Nicolls 1998). La recurrencia de administraciones de esta naturaleza ha provocado una hecatombe sociopolítica, económica y ambiental (Stotzky 1997). Haití es el país más pobre 
de las Américas. De acuerdo al Programa Naciones Unidas para el Desarrollo (PNUD), Haití tiene el índice de desarrollo humano más precario de la región y uno de los más bajos del mundo (Tabla 1).

Tabla 1: $\quad$ Comparación Índice de Desarrollo Humano

\begin{tabular}{lcccc}
\hline Índice de Desarrollo Humano & Haití & $\begin{array}{c}\text { República } \\
\text { Dominicana }\end{array}$ & Costa Rica & Nicaragua \\
\hline Alfabetismo & $48,1^{*}$ & 87 & 94,9 & 76,7 \\
Esperanza de Vida & 51,5 & 67,5 & 78,3 & 69,5 \\
PIB/per cápita (ppp) & $\$ 1.844$ & $\$ 7.499$ & $\$ 9.481$ & $\$ 3.634$ \\
Mortalidad Infantil menores de cinco años & $117 / 1.000$ & $32 / 1.000$ & $13 / 1.000$ & $38 / 1.000$ \\
Acceso a Agua Potable & $54 \%$ & $95 \%$ & $97 \%$ & $79 \%$ \\
Desnutrición & $47 \%$ & $27 \%$ & $4 \%$ & $27 \%$ \\
\hline
\end{tabular}

* Estudio parcial de UNESCO correspondiente al 2005. Fuente: Programa de Naciones Unidas para el Desarrollo.

Las condiciones de seguridad para la población son extremadamente precarias. Los ciudadanos están totalmente desprotegidos y son victimizados por bandas criminales que operan con total impunidad, tanto en áreas rurales como urbanas. En gran parte de las zonas rurales de Haití, la inexistencia de presencia estatal es llenada por caciques locales que apoyados por antisociales -muchas veces soldados del desmovilizado ejército y policías dados de baja- se han convertido en las autoridades de facto. En las ciudades, en tanto, miembros del crimen organizado, en particular bandas vinculadas al narcotráfico, controlan diversas zonas sin que intervenga la débil y corrupta policía. Esta situación se ve agravada por una severa crisis económica caracterizada por una enorme cesantía y desabestecimiento alimenticio en donde la población desesperada ve en el crimen la única posibilidad de protección y ascenso social (HRW 2005; BBC 2005a; Keen 1998).

El último episodio de la interminable crisis que afecta a Haití se desató en el 2004. En febrero de ese año rebeldes del Front révolutionnaire pour l'avancement et le progrès haïtien (FRAPH), comenzaron una ofensiva militar desde la ciudad norteña de Gonaives y que terminó con el derrocamiento del segundo gobierno de Jean-Bertrand Aristide. Aristide, el primer presidente democráticamente elegido en la historia de Haití, había tenido que huir del país en 1990 unos meses después de asumir la presidencia tras ser derrocado por un golpe militar encabezado por el general Raoul Cedrás, quien presidió un gobierno corrupto y autoritario en el que recreó muchos aspectos del gobierno de los Duvalier. En 1994, tras la remoción del gobierno de facto encabezado por el general Raoul Cedrás y la llegada de una fuerza multinacional de paz de Naciones Unidas encabezada por Estados Unidos, Aristide regresó a Haití para finalizar su mandato. Aristide fue reemplazado tras elección popular por la primera administración de René Préval (1995-2000). Aristide resultó electo presidente nuevamente en el 2000. Su segundo término, sin embargo, estuvo caracterizado por desaciertos en el manejo económico, corrupción generalizada y gran represión en contra de las fuerzas de oposición política. 
Es en ese contexto que se produjo la rebelión de FRAPH, grupo compuesto por efectivos del depuesto gobierno militar del general Cedrás. La ofensiva militar del FRAPH desató una violenta confrontación y sembró el caos en el país: mientras los rebeldes avanzaban sobre Puerto Príncipe desde su base en la ciudad nortina de Gonaives aterrorizando y saqueando, las fuerzas leales del presidente Aristide apoyadas por grupos paramilitares -los Chimères-, cometieron toda clase de excesos en contra de la población civil. El sistema penal del país, mientras tanto, colapsó tras la toma y destrucción de la mayoría de las prisiones, lo que derivó en la evasión masiva de la población penal. La situación de violencia generalizada provocada por el conflicto armado interno, y el concomitante quiebre de la precaria institucionalidad existente provocó una emergencia humanitaria compleja de vastas proporciones (CICR 2004; Kreps 2007).

La salida de Aristide al exilio en febrero del 2004 derivó en la instauración de un gobierno interino dirigido por Boniface Alexandre. La administración de Alexandre fue encabezada por su primer ministro, Gérard Latortue, la que se basó en el apoyo de la Misión de Estabilización de las Naciones Unidas en Haití (MINUSTAH). Esta última intervino para poner orden a la caótica situación del país y ayudó a restaurar un semblante de orden al país. Aún así, y a pesar de la presencia de una fuerza multilateral de paz compuesta por un contingente importante de soldados y policías, el Estado de Haití continúa siendo crítico. Organizaciones de derechos humanos indican que asesinatos con motivación política, detenciones arbitrarias, secuestros, ejecuciones extrajudiciales, asesinatos de civiles, violación, amenazas y extorsión son perpetrados con regularidad por agentes del Estado y miembros de grupos armados no estatales que operan con total impunidad. Periodistas, activistas de derechos humanos, trabajadores de organizaciones humanitarias e incluso cascos azules de la ONU han sido víctimas de la violencia (HRW 2007).

Las abismales condiciones de seguridad y la ausencia de perspectivas económicas para la población han provocado fuertes presiones migratorias. La mayoría de la diáspora haitiana se encuentra en República Dominicana; existen otras comunidades importantes en Estados Unidos (en Florida) y en Canadá (Québec). Esta migración está compuesta tanto por víctimas de desplazamiento forzado producto de la violencia, como de migrantes económicos (CIDH 2000; párrafos 48 y 49; Helton 2002: 86-102; Castles y Miller 2003: 150-1). Sendos desastres naturales como la reciente tormenta tropical Noel profundizaron los problemas del país, en particular el de algunas comunidades como Cabaret al norte de la capital, Puerto Príncipe. Noel, que azotó el país en octubre de 2007, provocó graves inundaciones, decenas de muertes y dejó alrededor de 6 mil damnificados. El gobierno estimó los daños en US\$1,5 millones (France Press 2007). Los desastres naturales que afectan el país no sólo perjudican la precaria infraestructura, sino que a raíz de la erosión del suelo y la deforestación, tienden arrasar hectáreas de cultivos y a provocar devastadoras inundaciones (MSF 2004).

\section{CARACTERÍSTICAS DEL SISTEMA POLÍTICO HAITIANO}

Haití es un sistema presidencialista de carácter híbrido en el cual el presidente es el jefe de Estado y el Primer Ministro el jefe de Gobierno. Dada la historia del país, en particular a lo que se refiere a los abusos de autoridad por parte del Jefe de Estado, el actual sistema 
político (reflejado en la Constitución de 1987) se caracteriza por buscar el equilibrio de poderes y por contener cláusulas específicas que buscan limitar el Poder del Ejecutivo. El presidente elige a su primer ministro, quien tiene que ser miembro del partido mayoritario en el Parlamento. La decisión del presidente tiene que ser ratificada por el Parlamento. El presidente tiene la prerrogativa de remover al Primer Ministro. El Parlamento, por su parte, puede removerlo también a través de un voto de no confianza que se concreta por mayoría simple en ambas cámaras. El gabinete es elegido por el Primer Ministro en consulta con el presidente y con la aprobación del Parlamento (Constitución de Haití 1987).

Las cámaras están compuestas por 30 (Alta) y 99 (Baja) representantes respectivamente. Los miembros del Senado son elegidos por voto popular y sirven por períodos de 6 años. Un tercio de la cámara se renueva cada dos años. Los miembros de la cámara baja, en tanto, sirven por períodos de cuatro años. El Parlamento tiene la prerrogativa de impugnar al Jefe de Estado, el Jefe de Gobierno o bien los ministros de Estado si reúne dos tercios de los votos en ambas cámaras. El Senado, por su parte, puede sugerir la nominación de los jueces de la Corte Suprema. Tanto el Poder Ejecutivo como el Poder Legislativo (ambas cámaras) pueden enviar una ley al Parlamento para su discusión y posterior promulgación. Sólo el Ejecutivo, sin embargo, puede enviar leyes relacionadas con el presupuesto, recaudación de impuestos y otros mecanismos de recolección de fondos fiscales (Constitución de Haití 1987).

Un punto muy importante para entender la política interna haitiana dice relación con la existencia de corrientes corporativistas en el sistema social y político. Por corporativismo nos referimos a un sistema mediante el cual el gobierno, a través de estructuras relativamente autónomas, busca imponer sobre la sociedad un sistema de representación de intereses basado en un pluralismo restringido que se caracteriza por la capacidad del Estado de controlar a las organizaciones sociales y a los grupos de presión (Malloy 1977:4). Desde la independencia del país en el siglo XIX, la sociedad haitiana y su sistema político se han caracterizado por la existencia de lo que Stotzky denomina corporativismo bifrontal. Este sistema le permite al Estado controlar a diferentes sectores de la sociedad civil, pero, al mismo tiempo, posibilita a varios sectores claves de la sociedad el establecimiento de bolsones de privilegio y de dominación dentro de la misma estructura del Estado. Entre estos grupos, destaca Stotzky, es posible mencionar a la Iglesia, las Fuerzas Armadas, la elite económica, grupos empresariales y algunas otras organizaciones cívicas (Stotzky 1997:88).

\section{EL PRIMER AÑO DE LA SEGUNDA ADMINISTRACIÓN DE RENÉ PRÉVAL}

El actual presidente de Haití, René Préval comenzó su segundo mandato tras imponerse en los comicios de febrero de 2006 con el 51\% de los votos. Su partido, Lespwa (esperanza) logró la primera mayoría en ambas cámaras. De los treinta asientos del Senado obtuvo doce (36\%) mientras en la cámara de representantes sacó 23 diputados (22\%). Los otros partidos con mayor votación corresponden a Fusión de Partidos Social Demócratas, la Alianza Democrática (Alyans) y los remanentes del partido Fanmi Lavalas, cercano al ex presidente Aristide, quien hoy se encuentra exiliado en la República de Sudáfrica. Este partido, sin embargo, se encuentra desintegrado producto de fuertes luchas intestinas (Tabla 2). 
Tabla 2: Número de Congresistas Cámara Alta y Baja

\begin{tabular}{lcc}
\hline PARTIDOS & $\begin{array}{c}\text { Número } \\
\text { Congresistas }\end{array}$ & $\begin{array}{c}\text { Número } \\
\text { Congresistas } \\
\text { CB }\end{array}$ \\
\hline Lespwa & 12 & 23 \\
Fusion des Sociaux-Democrates Haitienne & 4 & 17 \\
Alliance Democratique & 1 & 11 \\
Famni Lavalas & 3 & 6 \\
Organisation du Peuple en Lutte & 4 & 10 \\
Latibonit Ann Aksyon & 2 & 4 \\
Pou Nou Tout & 2 & 0 \\
Union Nationale Chrétuenn por la Rconstruction d'Haiti & 2 & 6 \\
Mobilisation pour le Progrès d'Haiti & 0 & 4 \\
Rassemblement des Dèmocrats Nationaux Progressiste d'Haiti & 0 & 4 \\
Mouvement Chrètien pour Batir unue Nouvelle Haiti & 0 & 3 \\
Konbit pou Bati Ayiti & 0 & 3 \\
Front pour la Reconstruction Nationale & 0 & 2 \\
Justice pour la Paix et le Dévelopmenr Nacional & 0 & 1 \\
Mouvement Dèmocratic et renovateur d'Haiti & 0 & 1 \\
Mouvement pour la Reconstruuction Nationale & 0 & 1 \\
Ted Ansamn & 0 & 1 \\
Parti Liberal Haitiene & 0 & 99 \\
TOTAL & 30 & \\
\hline
\end{tabular}

Fuente: International Crisis Group.

Las elecciones organizadas por el gobierno interino del presidente Boniface Alexandre fueron controversiales. Además de ser pospuestas por dificultades de todo tipo cuatro veces, las elecciones tuvieron un comienzo caótico y se caracterizaron por problemas e irregularidades. Hubo acusaciones de fraude, manipulación electoral y falta de transparencia. La campaña de Préval acusó al gobierno interino de obstaculizar su triunfo a través de maniobras ilícitas por parte del Consejo Electoral (CE). Durante el conteo de votos, el CE intentó invalidar votos de Préval, algo que impedía que este ganase un porcentaje superior al $50 \%$ y lo privaba de ganar en primera vuelta. Tras días de incertidumbre y gran presión internacional y doméstica, la crisis se resolvió cuando el CE declaró ganador a Préval con $51 \%$. Los observadores internacionales, incluidas Naciones Unidas y la Organización de Estados Americanos (OEA) avalaron el proceso (Esquenazi 2006).

Tanto la ONU como la OEA reconocieron la existencia de problemas en el proceso eleccionario pero enfatizaron que, dada la profunda crisis que afectaba a Haití, la realización de elecciones era en sí misma un gran triunfo sobre la base de la cual el país podía comenzar su recuperación (BBC 2006a). En un lúcido comentario al respecto se arguye que no quedaba claro si los problemas de la elección se debieron a una conspiración, a simple 
incompetencia o a una combinación de ambas, pero que no se podía esperar mucho más del proceso en un Estado semicolapsado (Economist 2006).

Más allá de la realización de un proceso electoral relativamente exitoso que logró sortear la crisis que azolaba al país, el proceso en sí mismo refleja la debilidad estructural del país. La organización de la elección fue posible gracias a Naciones Unidas y una vez que se suscitaron problemas fue la comunidad internacional la que medió para superar el impasse derivado de la decisión del CE de no validar miles de votos del partido de Préval. Esta situación es preocupante, ya que no avizora la posibilidad de realizar elecciones sin la intervención internacional, lo que evidentemente refleja grados importante de dependencia.

Préval heredó un país devastado, políticamente fracturado, con una economía moribunda y graves problemas de seguridad, sobre todo tras el desbande de la policía y el ejército. Durante sus primeros seis meses y apoyado por los efectivos de MINUSTAH logró restablecer paulatinamente ciertos grados de estabilidad y promover una moderada reactivación económica. Este proceso se profundizó durante el 2007. Desde que asumió el mando en mayo del 2006, Préval ha mostrado sus habilidades y gran astucia política. Consciente de que su mandato electoral era débil, Préval ha gobernado en alianza con los otros dos partidos de vasta votación y arraigo, Fusión de Partidos Social Demócratas y la Alianza Democrática (Alyans). Préval, asimismo, ha gozado del apoyo esporádico de ciertos sectores del partido Fanmi Lavalas. El pragmatismo de Préval y su habilidad para leer adecuadamente el ambiente político y que los desafíos del país necesitaban de un programa inclusivo se refleja en la nominación de un gabinete pluripartidista encabezado por su Primer Ministro Jacques-Edouard Alexis. Los 18 ministros que eligió pertenecían a seis partidos distintos incluidos Fanmi Lavalas (BBC 2006c) (Tabla 3). La nominación del gabinete fue aprobada por amplio margen en las cámaras. Gracias a su estrategia, Préval allanó el camino para llevar a cabo su programa de gobierno sin tener que depender de decretos presidenciales, como ocurrió en su primer gobierno (InterAmerican Dialogue 2007).

Después de 18 meses de relativa calma, sin embargo, en octubre de 2007 el clima de concordia se rompió tras la propuesta de Préval de modificar la Constitución de 1987. Sus declaraciones, en particular la falta de especificidad respecto a qué elementos de la Constitución considera necesario reformar, generaron toda clase de especulaciones sobre su supuesta intención de reformar la cláusula relativa a la reelección y así prorrogar su estadía en el poder. Préval argumenta que la Constitución creada tras la caída del gobierno de Jean Claude Duvalier se centra demasiado en generar mecanismos de supervisión al Poder Ejecutivo, a modo de evitar potenciales abusos y que, por tanto, hace excesivamente difícil gobernar al país. Aunque Préval negó insistentemente que su intención sea buscar la reelección, la mera proposición de reformar la Constitución y las insinuaciones relativas a la necesidad de dar más libertad al Poder Ejecutivo para realizar su programa de gobierno polarizó el frágil sistema político haitiano, exponiendo la fragilidad del gobierno de Préval. Por otro lado, el aplazamiento de las elecciones de parte de la Cámara Alta, así como propuestas para disolver el CE provisional, también generaron fuertes tensiones. Si bien las propuestas de Préval no debieran a priori ser tomadas como una manifestación de su deseo de preservarse en el poder, éstas debilitan el proyecto del presidente al erosionar 
Tabla 3: Composición del Gabinete Ministerial

\begin{tabular}{ll}
\hline \multicolumn{1}{c}{ Ministro } & \multicolumn{1}{c}{ Cartera } \\
\hline Jacques-Edouard Alexis & Primer Ministro \\
Antonio Phillipe Joseph & Relaciones Exteriores \\
Gustave Faubert & Economía y Finanzas \\
Gary Lissade & Justicia \\
Claude Menard & Interior \\
Marc Louis Bazin & Cooperación Externa \\
Sebastien Hilaire & Agricultura \\
Gaston George Merisier & Educación \\
Ernst Laraque & Trabajo Publico y Comunicación \\
Eude Saint-Preux Craan & Asuntos Sociales \\
Martine Deverson & Turismo \\
Webster Pierre & Medio Ambiente \\
Guy Paul & Cultura y Comunicación \\
Ginette Riviere Lubin & Asuntos de la Mujer \\
Henry Claud Voltaire & Haitianos residentes en el Exterior \\
Jocelerme Privert & Secretaria de Estado de Finanzas \\
Maryse Guyteau & Secretaria de Estado para la Alfabetizacion \\
Hermane Nau & Secretaria de Estado pa la Juventud y el Deporte \\
\hline
\end{tabular}

Fuente: Embajada de Haití en Washington.

su legitimidad y su vocación de compromiso y por ende ponen en peligro los pequeños logros de su administración. Tal como afirman privadamente diplomáticos apostados en Haití, dada la gravedad de los problemas que aquejan al país, la discusión sobre reformas a la Constitución es una distracción innecesaria que Haití no puede darse el lujo de tener (The Miami Herald 2007).

El principal objetivo de Préval una vez que asumió el poder fue el de restaurar las condiciones de seguridad en el país. Durante el 2007, gracias a un esfuerzo mancomunado entre el gobierno y MINUSTAH, las condiciones de seguridad mejoraron substancialmente, sobre todo en la capital. Como se indicó anteriormente, durante la aguda crisis que provocó la salida de Aristide en el 2004, el país descendió a una situación de anarquía hobbsiana en la que facciones armadas se disputaron el poder violentamente sembrando el terror en la población. El caos se gestó en gran medida por el desbande total del ejército y la policía que, lejos de controlar a los grupos armados, tomaron partido por éstos (International Crisis Group 2006: 9-11).

Si bien el país ha retornado a condiciones más aceptables, la seguridad sigue siendo crítica. Un problema gravísimo que afecta al país es la presencia del crimen organizado. Por un lado, existen bandas de narcotráfico que utilizan Haití como punto de tránsito de droga que va rumbo a Estados Unidos. Por otro, persisten una serie de bandas que se dedican al plagio/secuestro de personas. Estos grupos generalmente tienen aspiraciones 
y vínculos con sectores políticos y mantienen el control en vastos sectores de los barrios marginales de Puerto Príncipe y otras ciudades. El problema principal es la inexistencia de presencia estatal: sin contingentes policiales que vigilen e impongan orden, ni instituciones que administren justicia y ante la ausencia casi total de servicios sociales y empleo, las condiciones e incentivos para la comisión de delitos y abusos es enorme. En muchos sectores rurales grupos de particulares, en general bandas criminales muchas veces compuestas por ex soldados del ejército y policías dados de baja, se han convertido en las autoridades de facto. Como resultado, se ha generado un ambiente caracterizado por altos niveles de impunidad y donde la población civil está totalmente indefensa ante los abusos de estos grupos (ICG 2006: 6; ICG 2004; HRW 2007).

En materia de seguridad, la situación de Cité Soleil resulta particularmente sensible. En este barrio marginal de la capital haitiana habitada por entre 200-300 mil personas operaban a comienzos de 2007 al menos 34 bandas delictivas altamente organizadas. Estos grupos que se distribuían en los 32 distritos de Cité Soleil competían de manera violenta por la supremacía a efectos de poder llevar a cabo negocios ilícitos, como tráfico de estupefacientes, hurtos, robo de automóviles, extorsión, secuestros y tráfico de armas. La naturaleza y composición de estos grupos varía entre organisations populairs (OPs), grupos armados con vínculos al ex presidente Aristide (chimères), grupos de crimen organizado, vinculados a ex agentes de la policía, crimen organizado transnacional y bandas compuestas por ex miembros de las Fuerzas Armadas haitianas. Muchos antisociales trabajan de forma independiente como gatilleros e informantes de estos grupos. Delincuentes comunes (zenglendos), prófugos de la justicia, personas deportadas por crímenes desde EUA y Canadá y agentes corruptos de la policía también participan en las actividades delictivas. Por otro lado, la prevalencia de delitos sexuales es particularmente alarmante (ICG 2006: 1-4; ICG 2007a; The Economist 2004).

Durante los primeros seis meses del mandato de Préval y a pesar de la presencia de un contingente de tropas de MINUSTAH la situación de seguridad continuó siendo crítica.

Si bien los índices de violencia se concentran en las principales urbes del país como Puerto Príncipe, Cap-Haïtien, St. Marc, Gonaïves, Jacmel, Les Cayes, Port Salut, Port au Paix, entre otras, la situación en materia de seguridad en zonas rurales prosiguió siendo grave.

El plan para mejorar la seguridad del gobierno de Préval se basó en dos pilares fundamentales. En primer lugar, a través de los contingentes de MINUSTAH, se inició un intenso proceso de neutralización de las facciones armadas. Parte importante de este trabajo incluyó un programa de desarme. Por otro lado, miembros de MINUSTAH han desarrollado un agresivo programa de entrenamiento y capacitación para la Policía Nacional Haitiana (PNH), una nueva policía civil que reemplazó la antigua policía militar haitiana.

La estrategia se articuló en un programa denominado Desarme, Desmobilización y Reintegración lanzado en el 2006 pero que continuó a toda marcha el 2007, y que buscaba, al menos en principio, una solución integral y comprehensiva al problema de la delincuencia. Esto se lleva a cabo al combinar acciones punitivas con incentivos, en particular facilidades para la reinserción de miembros de las bandas armadas a la sociedad haitiana. 
Durante noviembre y diciembre del 2006 los niveles de inseguridad, incluidos ataques a fuerzas de MINUSTAH, fueron altísimos. En noviembre del 2006 dos soldados jordanos de MINUSTAH murieron producto de ataques mientras patrullaban Cité Soleil. Otro problema gravísimo en materia de seguridad dice relación con los secuestros. La extrema violencia y su carácter masivo han sembrado el terror en la población haitiana, en particular la de Puerto Príncipe, donde se concentran la mayoría de los casos. Los secuestros llegaron a su cenit en diciembre del 2006. Según cifras de MINUSTAH, hubo 129 casos sólo ese mes. Algunos plagios fueron particularmente brutales, como el asesinato de la estudiante Farah Dessources y el de un niño de seis años, incluso después que el rescate había sido entregado (ICG 2007a:2). A partir del mes de noviembre de 2007, una nueva oleada de secuestros de niños azotó a Haití en Delmas y Puerto Príncipe, la que se ha extendido hasta los primeros meses de 2008, generando condenas por parte de amplios sectores de la población y de organizaciones como el Fondo para la Infancia de la ONU (UNICEF 2008). Si bien los índices fueron inferiores al período más activo del 2006, los plagios generaron preocupación y revelan que la situación de seguridad sigue siendo precaria (Prensa Latina 2007).

Numerosos miembros del Parlamento haitiano, organizaciones de la sociedad civil haitianas, así como organizaciones de derechos humanos criticaron la actitud del gobierno de priorizar el diálogo con líderes de las facciones armadas. En opinión de los críticos, basar el programa de desarme en el diálogo con grupos delictivos promueve condiciones generales de impunidad. Estas condiciones, sostienen, son nocivas para el país (Amnistía Internacional 2007; The Economist 2007).

A partir de diciembre del 2006, efectivos de MINUSTAH y miembros de la PNH llevaron a cabo una serie de operaciones en barrios marginales de Puerto Príncipe, en particular Cité Soleil y Martissant, y cuyo objetivo era desbaratar bandas de criminales organizados. Gracias a trabajo de inteligencia y al aumento de la vigilancia a través de retenes especiales, MINUSTAH y efectivos de la PNH pacificaron vastas zonas marginales, arrestando o bien abatiendo en combate a líderes y miembros de estas bandas. Sólo entre enero y julio del 2007, más de 750 miembros de diversas bandas criminales fueron arrestados o murieron en enfrentamientos con tropas MINUSTAH o efectivos de la PNH. Informes de las autoridades y de MINUSTAH indican que muchas de las bandas se sumergieron y escondieron sus armas para evitar ser desbandadas por las fuerzas de MINUSTAH (InterAmerican Dialogue 2007:2; ICG 2007a:2). Parte del éxito de estas operaciones es el resultado de un mejoramiento substancial en los niveles de cooperación entre Naciones Unidas y las autoridades haitianas (Interamerican Dialogue 2007:2; The Guardian 2007).

Las operaciones conjuntas de las fuerzas de Naciones Unidas y la PNH han mejorado la situación de seguridad, sobre todo en Puerto Príncipe. Asaltos, ataques y secuestros han disminuido tras el desmantelamiento de algunos de los grupos más violentos. El año 2005 se reportaron 634 secuestros en Haití, 488 el 2006 y 279 el 2007. Hasta el 15 de marzo de 2008 sumaban 83 secuestros reportados, lo que implica un repunte respecto de 2007. El control de estos grupos además ha contribuido en parte al repunte económico del país: mejores condiciones de seguridad han posibilitado la apertura de los puertos y facilitado la distribución de productos y servicios; asimismo, ha disminuido la extorsión a los comerciantes e inversores. 
Medidas de seguridad por parte de las autoridades y MINUSTAH han sido complementadas por iniciativas de seguridad ciudadana por parte de la sociedad civil. Por ejemplo, las nuevas autoridades comunales de Cité Soleil encabezadas por el joven alcalde Wilson Louis del grupo independiente Pel et Picwa han coordinado algunos pequeños programas de seguridad ciudadana, pero estos han sido sólo parcialmente exitosos. La labor es tremendamente difícil dadas las necesidades urgentes y masivas de la comunidad y la falta de recursos para satisfacerlas (ICG 2007a:1-3; The Miami Herald 2007; InterAmerican Dialogue 2007:2).

Un punto delicado y que refleja que persisten problemas en relación a las condiciones de seguridad tiene que ver con un incremento significativo de los linchamientos a lo largo del país. Comunidades de ciudadanos han tomado la justicia en sus manos en diversas zonas y han linchado a presuntos delincuentes, en particular personas acusadas de participar en secuestros. Muchas víctimas han sido personas que han sido descubiertas en flagrancia o que han sido identificadas por la comunidad en la comisión de delitos (ICG 2007A:4; BBC 2007). La situación es preocupante porque el descontrol y ausencia de estructuras estatales pueden generar un incremento de acusaciones frívolas destinadas a saldar viejas rencillas, multiplicando así un ciclo de violencias y retaliación.

De este análisis somero resulta aparente que, más allá de algunos avances, las cuentas pendientes en materia de seguridad son formidables. En primer lugar, gran parte de las mejorías sólo se han materializado en Puerto Príncipe, por lo que en la inmensa mayoría del país las condiciones siguen siendo preocupantes, tanto en sectores rurales como urbanos. Durante 2007 y una vez controladas las bandas armadas más peligrosas, las fuerzas de MINUSTAH y la PNH se comenzaron a concentrar en el crimen organizado. Por sus recursos y resiliencia, empero, el combate a estos grupos promete ser muy difícil y extenuante. Otro desafío pendiente pero importantísimo en esta materia tiene que ver con la necesidad de depurar y profesionalizar la PNH. Cientos de miembros de la policía han sido dados de baja y muchos más están en proceso de investigación. Sin embargo, en la fuerza aún continúa un porcentaje no menor de individuos a quienes se acusa de corrupción y otras faltas graves. Mientras tanto la PNH se ha abocado a la selección y entrenamiento de nuevos reclutas. Otro tema delicado referente a la PNH es la reinserción social de los elementos dados de baja de la fuerza. La enorme mayoría de estas personas no han ido a juicio. En libertad pero en un contexto en el que no se han implementado programas que faciliten su reinserción a la vida civil, sobre todo con oportunidades laborales, muchos policías retomaron actividades criminales. Su entrenamiento y su conocimiento sobre procedimientos de las redes de información, los convierten en delincuentes mucho más difíciles de controlar (ICG 2007a: 4).

Si bien la seguridad ha mejorado, resulta muy preocupante el grado de dependencia de la MINUSTAH para lograr este objetivo. Gran parte de los avances en materia de seguridad se han gestado gracias a la presencia de las tropas de Mantenimiento de la Paz. Respecto a la PNH, si bien ha habido avances, el grado de desarrollo institucional todavía es incipiente, por decir lo menos, por lo que parece aventurado pensar que las autoridades haitianas podrían por sí mismas mantener -ni mucho menos mejorar- 
la situación general de seguridad en el país. Por último, si MINUSTAH que maneja un presupuesto ampliamente superior al del Estado haitiano y tiene personal militar y policial especializado, ha obtenido una victoria pírrica en materia de seguridad, la prognosis una vez que MINUSTAH se retire no es de las mejores. En otras palabras, la seguridad actual es en parte el resultado de la presencia de tropas extranjeras por lo que los niveles de dependencia son altos.

La situación en materia de derechos humanos en Haití no mejoró substantivamente durante el reciente año. Acusaciones muy serias de violaciones a los derechos humanos persisten en contra de agentes estatales, en particular la PNH. A ésta se acusa de la comisión de diversos abusos, entre ellos, el uso excesivo de fuerza, arrestos arbitrarios (redadas), asesinatos y de extraer confesiones bajo tortura. Por otro lado, en Haití persisten problemas muy graves de acceso y administración de justicia. El sistema de justicia padece problemas estructurales muy serios. A este respecto, se combina la enorme falta de recursos, capacitación inadecuada de los funcionarios y altísimos grados de corrupción. Como consecuencia, los crímenes no son investigados, ni los responsables juzgados. Las pocas investigaciones y juicios que se llevan a cabo se caracterizan por serias deficiencias que no garantizan condiciones mínimas de debido proceso. En Haití cientos de personas están privadas de libertad sin cargos en su contra y no cuentan con acceso a defensa. Esto genera condiciones generalizadas de impunidad y abuso, tanto a inocentes como a personas que han violado la ley. Las condiciones para las personas privadas de libertad son particularmente inhumanas y degradantes. En la mayoría de los penales del país los reclusos subsisten gracias a los recursos que envían sus familiares, mientras que las condiciones de salubridad, hacinamiento, acceso a servicios y violencia en las instituciones son sencillamente horrorosas (HRW 2007, IACHR 2007; Oficina del Alto Comisionado de las Naciones Unidas 2007).

Otro problema importante es la politización del sistema judicial. La tradición de la política haitiana en la que el Poder Ejecutivo interfiere en decisiones del Poder Judicial a efectos de reforzar sus posiciones y debilitar a sus detractores y enemigos persiste. De acuerdo a varias fuentes, en el país existen más de un centenar de prisioneros políticos. Muchos corresponden a personas acusadas durante administraciones anteriores y cuyos casos no han sido debidamente investigados; otros corresponden a militantes del partido Levalas acusados de diversos delitos. Durante el 2007 la violencia por motivos políticos continuó en contra de militantes de diversos partidos, tanto de gobierno como opositores, defensores de derechos humanos, activistas comunitarios, periodistas y abogados, entre otros. Particularmente graves son algunos asesinatos colectivos e indiscriminados llevados a cabo por grupos armados irregulares (HRW 2007; ICG 2007a:12).

La violencia de género es otro elemento extremadamente preocupante. Mujeres y niñas son regularmente hostigadas, torturadas, violadas y muchas veces asesinadas por grupos irregulares sin que las autoridades intervengan. Muchas mujeres son forzadas a prodigar servicios sexuales a miembros de bandas organizadas, exponiéndose a la transmisión de enfermedades como HIV y Hepatitis B. La violación de los derechos fundamentales de niños y niñas también es un problema gravísimo. En los últimos meses y tras la finalización de los operativos de seguridad se ha apreciado un incremento fuerte en la comisión de delitos 
sexuales como violaciones en contra de mujeres menores de edad en sectores marginales de Puerto Príncipe como Cité Soleil. Otro problema grave dice relación con los menores de edad. Los menores son víctimas de abusos sexuales, reclutamiento forzado. Muchos grupos armados utilizan a niños como escudos humanos durante operaciones militares o bien en la comisión de robos y otros delitos. La violación de derechos económicos, sociales y culturales también es masiva y evidente. Los problemas más urgentes incluyen acceso a la educación, salud, vivienda y a un medioambiente limpio (ICG 2007A: 1-5; Comisión Interamericana de Derechos Humanos 2007; HRW 2007). Según encuestas realizadas el 2006 por el Latin American Public Opinion Project (LAPOP), en Haití la gente expresa grados significativos de desconfianza en las instituciones formales, temor ante la prevalente delincuencia y rechazo a la corrupción existente en el país. Muchas oficinas de gobierno, incluidos ministerios, son sólo fachadas completamente ineficaces debido a la falta de recursos y de capacidades básicas de acción ejecutiva. El grado de victimización no es el de los más altos de la región según LAPOP, pero esto puede deberse a un subregistro de los casos. Muchos de los crímenes reportados son graves (Altman y Luna 2007).

\section{REACTIVACIÓN ECONÓMICA}

Probablemente uno de los elementos más positivos del último año en Haití ha sido la robusta reactivación económica que experimentó el país. Después de años de contracción o crecimiento anémico de la economía, Haití creció 3\% durante 2007, una cifra positiva, aunque por debajo de las expectativas del gobierno que pronosticó un crecimiento del $4 \%$. El crecimiento se vio afectado por la reticencia de inversores de entrar en el mercado haitiano dada las condiciones de inestabilidad y la ausencia de seguridad jurídica, así como por factores externos incluidos los altos precios del petróleo y la energía (EIU 2007). Aún así la economía se expandió empujada por aportes internacionales como petróleo subsidiado desde Venezuela y en especial por la presencia de la cooperación internacional. Por otro lado, mejores condiciones de seguridad permitieron la reactivación de algunos sectores de exportación relevantes que se habían visto afectados por la reciente crisis, en particular el sector textil y agrícola y algunos servicios. El sector textil lideró el repunte gracias al incremento de exportación a Estados Unidos derivado de la Haitian Hemispheric Opportunity Through Partnership Encouragment (HOPE), iniciativa que se hizo efectiva en junio y que exime de tarifas a los productos textiles haitianos que ingresan al mercado estadounidense (EIU 2007; ICG 2007a:8; The Economist 2007b). Por otro lado, las reservas del país y la recaudación tributaria también crecieron (ICG 2007a: i). La reducción substancial del servicio a la deuda por parte del Fondo Monetario Internacional (FMI), gracias a la iniciativa multilateral de reducción de la deuda externa a los países altamente endeudados, contribuyó a liberar fondos para promover una serie de programas de empleo y desarrollo de infraestructura que también favorecieron el crecimiento económico. Si bien el crecimiento es importante, es modesto si se toma en cuenta que en los últimos 40 años el ingreso per cápita del país se contrajo alrededor del 45 por ciento (Banco Mundial 2007).

El gobierno también tuvo una exitosa política de control de la inflación, que llegó a 8\% durante 2007. Condiciones climáticas más favorables que en temporadas anteriores, la 
apreciación del tipo de cambio y una política fiscal y monetaria disciplinada contribuyeron a mantener la inflación bajo control, aun cuando factores como la llegada de remesas de ciudadanos haitianos en el exterior, la expansión del crédito y la ayuda económica generaron presiones inflacionarias. Esos factores se espera que continúen presentes, los que sumados a la crisis alimenticia y a la persistente alza de precios de la energía, pueden hacer subir la inflación en el futuro cercano. La balanza de pagos, por otro lado, presenta problemas medulares. Una balanza comercial deficitaria refleja la alta dependencia del país de las exportaciones y sus dificultades para colocar sus productos en los mercados internacionales. Un problema relevante a este respecto es el descenso de la producción agrícola derivada de una combinación entre degradación ambiental y la falta de inversión, algo que unido al crecimiento poblacional afecta la dependencia foránea de la economía. Por otro lado, la falta de inversión extranjera ahonda el déficit de la cuenta corriente e impacta negativamente en la balanza de pagos (EIU 2007).

Un punto crítico tiene que ver con la generación de empleo. Si bien en el último año algunos sectores de la economía (como el textil) han generado nuevas plazas de trabajo y el gobierno en conjunto con países donantes ha desarrollado planes de construcción de infraestructura y reactivación económica, la falta de empleo continúa afectando al país. Un porcentaje bajo de los haitianos tiene empleo en el sector formal; la gran mayoría subsiste precariamente vendiendo servicios y productos en la economía informal. Esta situación se ve magnificada por severos índices de pobreza y analfabetismo. Como consecuencia, en Haití decenas de miles de personas no cuentan con las condiciones mínimas para asegurar su subsistencia ni tampoco acceso a servicios sociales básicos (vivienda, salud, educación, acceso a agua potable o transporte público) (HRW 2007; ICG 2007a). (Cuadro 3). La combinación entre condiciones de pobreza estructural y una economía precaria que no ofrece oportunidades de trabajo ni posibilidades de ascenso social y la existencia de organizaciones delictuales que necesitan mano de obra, genera incentivos para que muchas personas, sobre todo jóvenes de los sectores marginales, nutran a los grupos delictivos. De este modo se construye y alimenta un ciclo perverso entre falta de oportunidades y pobreza, por una lado, y condiciones de inseguridad, por el otro, que no hace más que reforzar los problemas económicos del país.

Al igual que con la situación general de seguridad, en materia económica la dependencia haitiana de la asistencia internacional es profunda. La existencia de inversión y de productos de consumo está íntimamente ligada a la presencia de la cooperación internacional, en particular el contingente de MINUSTAH que consumen bienes y servicios. La presencia de la comunidad internacional actúa como un estímulo a la economía. La generación de empleo es otro elemento clave: aunque no hay datos precisos, un porcentaje bajo pero no menor de personas vende productos y otorga servicios a las fuerzas extranjeras, lo que ayuda a estimular el empleo. La presencia de tropas extranjeras, por otro lado, tiene un efecto positivo en materia de seguridad lo que redunda en un impacto positivo para la economía. La literatura sobre trabajo humanitario ha establecido la existencia de externalidades negativas derivadas de la presencia de la cooperación internacional, entre ellas, una exacerbación de la dependencia económica, lo que evidentemente perjudica el desarrollo de la economía local. Larry Minear comenta que en Haití la presencia de la cooperación internacional tuvo efectos perversos durante los años noventa al acentuar 
problemas relativos a la pobreza, ineficacia en los servicios y a la performance de la economía (2005: 63). Esta situación subsiste.

\section{EL ROL DE MINUSTAH}

Dada la delicada situación sociopolítica y económica que enfrenta Haití, cualquier análisis sobre la situación del país amerita examinar el rol que juegan las fuerzas de Mantenimiento de la Paz de la ONU. Como se indicó, la severa crisis política que sacudió al país y que derivó en un conflicto armado interno corto pero devastador gatilló la intervención de la comunidad internacional a través de MINUSTAH. En vista del colapso casi total de la ley y el orden en el país y de la creciente victimización de civiles producto del conflicto armado, el Consejo de Seguridad de la ONU aprobó mediante una resolución despachar la Fuerza Multinacional Interina para Haití (MIFH) (que luego mutaría de nombre a MINUSTAH). La iniciativa contó con el apoyo político de la Organización de Estados Americanos (OEA) y también de la Comunidad Económica del Caribe (CARICOM). MINUSTAH representa la séptima Operación para el Mantenimiento de la Paz que el sistema de Naciones Unidas despliega en Haití desde el fin de la Guerra Fría (Álvarez Veloso 2005:4). El Consejo de Seguridad le otorgó a MINUSTAH un mandato amplio que incluye: (i) restablecer la seguridad y estabilizar al país; (ii) apoyar el proceso político interno, la reconciliación nacional y restablecer el sistema constitucional democrático; y (iii) promover y garantizar el respeto por los derechos humanos (MINUSTAH 2007).

Después de un comienzo muy difícil en el que los progresos de la misión fueron más bien modestos, durante los últimos 22 meses MINUSTAH cumplió con algunos de sus objetivos primordiales. Dos puntos destacan en la labor de MINUSTAH: el mejoramiento de las condiciones de seguridad en el país y la realización de elecciones presidenciales que propiciaron la elección de René Préval.

Es indudable que la organización y posterior realización de las elecciones presidenciales de febrero del 2006 fue muy importante, más allá de los evidentes problemas y deficiencias del proceso que se reseñaron con anterioridad. MINUSTAH tiene cierta cuota de responsabilidad en los problemas que se suscitaron en las elecciones; las críticas en particular apuntan a su debilidad frente a la administración interina de Alexandre que mostró un sesgo evidente en contra del partido Levalas. Sin embargo, dados los enormes desafíos que revestía organizar un proceso en un país económicamente devastado, fraccionado políticamente, con condiciones de seguridad inexistentes y la casi nula institucionalidad, la mera materialización de un proceso representó un gran avance. Esta sensación se refuerza dado que el triunfo electoral de Préval es percibido por la inmensa mayoría del país como legítimo.

Durante 2007 y comienzos de 2008, como ya se indicó, MINUSTAH ha contribuido de forma mucho más contundente a la pacificación del país a través de su agresivo programa de desarme y neutralización de la bandas armadas y entrenamiento de la policía. Hoy en día el país es mucho más seguro y la población puede desplazarse con relativa tranquilidad. Hasta fines del 2006 y comienzos de 2007, el impacto de la MINUSTAH 
en las condiciones de seguridad no fue tan contundente, empero. Si bien la situación de violencia y descontrol generalizado que caracterizó los meses que antecedieron a la salida de Aristide se mitigó con la entrada de MINUSTAH, las condiciones de seguridad continuaron siendo críticas. Parte del problema derivó de que en un comienzo MINUSTAH estaba compuesta sólo por 3.000 efectivos. Como reflejo de su exiguo número, las fuerzas de MINUSTAH operaban de manera restrictiva y rara vez abandonan sus centros de operación (compounds). El impacto aumentó paulatinamente tras el arribo de más cascos azules en los meses previos a las elecciones (Ezquenazi 2006, ICG 2007a; Howland 2006: 466; ICG 2007a) (Tabla 4).

Tabla 4: $\quad$ Países participantes de MINUSTAH

\begin{tabular}{lc}
\hline Participantes de MINUSTAH & Efectivos Militares* $^{*}$ \\
\hline Brasil & 1.213 \\
Uruguay & 1.148 \\
Nepal & 1.109 \\
Sri Lanka & 960 \\
Jordania & 755 \\
Argentina & 562 \\
Chile & 501 \\
Bolivia & 216 \\
Perú & 210 \\
Filipinas & 157 \\
Guatemala & 119 \\
Ecuador & 67 \\
Paraguay & 31 \\
Canadá & 4 \\
Croacia & 4 \\
Estados Unidos & 3 \\
Francia & 2 \\
Pakistán & 1 \\
\hline
\end{tabular}

* La misión incluye además 2.092 policías. Fuente: MINUSTAH.

Más allá de sus éxitos, MINUSTAH tiene deficiencias que es preciso recalcar. Detractores de la misión sostienen que las condiciones de seguridad continúan siendo críticas y que los progresos se circunscriben casi exclusivamente a Puerto Príncipe. Por otro lado, indican que los éxitos en materia de seguridad han sido a expensas de la trasgresión de principios fundamentales del Derecho Internacional Humanitario (DIH) y del Derecho Internacional de los Derechos Humanos. Entre los puntos más sensibles se destaca el comportamiento del batallón Jordano basado en Cité Solelil y al que se acusa de uso excesivo y desproporcionado de la fuerza durante refriegas contra milicias y que resultaron en la muerte de decenas de civiles, así como acusaciones de abusos sexuales en contra del batallón de Sri Lanka este año (BBC 2007b). La misión de la ONU ni siquiera investigó los hechos, a pesar de la 
gravedad de las acusaciones. Otro punto crítico dice relación con los excesos cometidos por la $\mathrm{PNH}$, frente a los que no se han tomado medidas concretas por parte del gobierno, y sobre los cuales MINUSTAH no se ha pronunciado. Si bien existe un programa para purgar a agentes corruptos o implicados en violaciones de derechos humanos y del cual MINUSTAH es responsable, éste ha sido caracterizado por una excesiva leniencia. De modo más profundo, críticos apuntan a que la misión no ha incorporado de forma seria y honesta una matriz transversal de derechos humanos como guía de sus programas, a pesar que su mandato enfatiza la prioridad de avanzar en la materia (Reed 2006).

Otro punto muy contencioso es su mutismo frente a los sesgos políticos y excesos cometidos por el gobierno interino de Alexandre. De forma más general, críticos apuntan a que la misión no ha sido inclusiva y que no ha involucrado suficientemente a la sociedad civil haitiana. El diseño y consecución de los programas han sido caracterizados por paternalismo y una cierta opacidad, en la que el diálogo con la sociedad haitiana es reducido y sólo se remite a las administraciones de turno. Finalmente, detractores apuntan a que los éxitos han sido demasiado modestos si uno toma en cuenta que MINUSTAH maneja un presupuesto más grande que el Estado haitiano (Howland 2006; Reed 2006).

\section{CONCLUSIONES}

Más allá de ciertos progresos en el último año, la prognosis para el futuro de Haití no invita al optimismo. La fragilidad es la característica transversal del proceso haitiano. La situación política aunque estabilizada puede tornarse tormentosa tras la decisión de Préval de modificar la Constitución política, algo que genera tensiones no sólo con la oposición que teme que Préval busque eternizarse en el poder, sino en la misma coalición de gobierno. Si bien MINUSTAH ha contribuido a promover la seguridad del país, ésta sigue siendo precaria. El proceso de reconstrucción de la PNH está plagado de problemas y desafíos. El crecimiento económico y el acertado manejo económico del gobierno de Préval representan una luz de esperanza; sin embargo, reformar las condiciones estructurales de una economía altamente disfuncional representa un desafío considerable para asegurar tasas de crecimiento estables en el futuro.

Por último, un elemento preocupante es el alto grado de dependencia de Haití de la asistencia internacional. En este instante el país depende de la asistencia internacional en casi todas las esferas de su quehacer (economía, seguridad, desarrollo institucional y político). Si bien el compromiso de la comunidad internacional, en particular de los gobiernos de Canadá, Estados Unidos, y en menor medida de algunos estados latinoamericanos como Argentina, Brasil, Chile y Uruguay, es de momento robusta, no es realista esperar que se mantenga en el largo plazo. Grados de donor fatigue y compromisos en otros puntos conflictivos evidentemente propiciarán en algún momento el retiro de la presencia internacional. A ese nivel, la pregunta clave es si el desarrollo institucional haitiano será suficiente para mantener condiciones de estabilidad y, de forma más general, la viabilidad del país en un escenario post-MINUSTAH (Thompson 2007). En un reciente estudio el International Crisis Group destaca la importancia que reviste una mayor presencia de la diáspora haitiana para 
avanzar en las tareas pendientes del país (ICG 2007b). Un tema relevante es cómo Haití resolverá las presiones a las que es sometido por Venezuela y Estados Unidos, dos países que bregan por mantener y expandir su influencia sobre el país (Cariot 2007). Mientras se resuelve esta incógnita, lo seguro es que proseguirán las tribulaciones de Haití.

\section{REFERENCIAS}

Altman, David y Juan Pablo Luna 2007".Desafección cívica, polarización ideológica y calidad de la democracia: una introducción al Anuario Político de América Latina". Revista de Ciencia Política 27 ( $\left.\mathrm{N}^{\circ} \mathrm{EE}\right)$ : 3-28.

Amnistía Internacional. 2007. Amnesty International Report: Haiti. [En línea] <http:/ /www.amnesty.org/ es/region/americas/caribbean/hait\%C3\%AD>. [Consulta: 25 de octubre 2007].

Banco Mundial.2007. Haiti Country Brief. [En Línea] <http:/ / web.worldbank.org/WBSITE/EXTERNAL/ COUNTRIES/LACEXT/HAITIEXTN/0,,contentMDK:21040686 pagePK:141137 piPK:141127 the SitePK:338165,00.html>[Consulta: 28 de octubre 2007]

BBC. 2007a. UN Concerned at Haiti Lynchings. . [En línea] <http://news.bbc.co.uk/2/hi/americas/6920240. stm> [Consulta: 18 de octubre 2007]

2007b. Sri Lanka to Probe UN Sex Claims. [En línea] <http://news.bbc.co.uk/2/hi/south_ asia/7076284.stm> [Consulta: 18 de octubre 2007].

2006a. Préval Declared Haiti Poll Winner. http://news.bbc.co.uk/2/hi/americas/4718790.stm

2006b. Haití: La ONU no quiere esperar más. [En línea] <http://news.bbc.co.uk/hi/spanish/ latin_america/newsid_4619000/4619132.stm> [Consulta: 12 de noviembre 2007].

2006b. New Haitian Cabinet Takes Office. [En línea] <http://news.bbc.co.uk/2/hi/americas/5066694. stm> [Consulta: 17 de noviembre 2007].

2005a. Haiti's security dilemma. [En línea] <http://news.bbc.co.uk/1/hi/world/americas/4253808. stm. > [Consulta: 17 de octubre 2007].

Cariot, Jean-Michel . 2007. "Le president Préval pris entre Washington et Caracas". Le Monde 12 mai. p. 4 .

Castles, Stephen y Mark Miller. 2003. The Age of Migration (tercera edición). New York: The Guilford Press.

Chehabi, H.E. y Juan Linz. 1998. "A Theory of Sultanism: A Type of Nondemocratic Rule". En Sultanistic Regimes, editado por H.E. Chehabi y Juan Linz. Baltimore: Johns Hopkins University Press, 3-25

Comisión Interamericana de Derechos Humanos.2007. Informe Anual. Desarrollo de Derechos Humanos en la Región: Haití[En línea] <http:/ / www.iachr.org/annualrep/2006eng/Chap.4d.htm> [Consulta: 13 de octubre 2007].

2000. Segundo Informe de Progreso de Relatoría Especial de Trabajadores Migratorios y sus Familias. [En línea] <http://www.iachr.org/annualrep/2000eng/chap.6a.htm\#Hemispheric\%20 trends $>$ [Consulta: 13 de octubre 2007].

Comité Internacional de la Cruz Roja. 2004. Annual Report: Haití. [En línea] <http://www.icrc.org/ Web/Eng/siteeng0.nsf/htmlall/6D6GYA/\$FILE/icrc_ar_04_haiti.pdf?OpenElement>[Consulta: 12 de octubre 2007].

Constitution de la République d'Haïti.1987. [En línea] <http://pdba.georgetown.edu/Constitutions/ Haiti/haiti1987.html>[Consulta: 12 de diciembre 2007].

Ezquenazi, Patricia. 2006. "A Day of Dawning Peace in Haiti". Americas 58 (3): 8-11.

France Press. 2007. Death Toll Reaches 43 as Tropical Storm Noel Rages Across Caribbean. October 31.

Helton, Arthur. 2002. The Price of Indifference. Oxford: Oxford University Press.

Howland, Todd. 2006. "Peacekeeping and Conformity with Human Rights: How MINUSTAH Falls Short in Haiti". International Peacekeeping 13 (4): 462-476.

Human Rights Watch. 2007. Haiti: Events of 2006. [En línea] <http://hrw.org/englishwr2k7/docs/2007/01/11/ haiti14862.htm>[Consulta: 17 de octubre 2007]. 
Inter-American Dialogue. 2007. Haiti: Real Progress, Real Fragility. [En línea] <http:/ / www.thedialogue. org/page.cfm?pageID=115>[Consulta: 24 de noviembre 2007].

International Crisis Group.2007a. Consolidating Stability in Haiti. Latin America/Caribbean Report 21, 18 July [En línea] <http:/ / www.crisisgroup.org/home/index.cfm?id=4944\&l=1>[Consulta: 24 de septiembre 2007].

2007b. Peacebuilding in Haiti: Including Haitians From Abroad. Latin America/Caribbean Report 24, 14 December. [En línea] <http://www.crisisgroup.org/library/documents/24_peacebuilding_ in_haiti__including_haitians_from_abroad.pdf $>$ [Consulta: 30 de septiembre 2007].

2006. Haiti: Security and the Reintegration of the State. Latin America/Caribbean Report 12, 30 October. . [En línea] < http:/ / www.crisisgroup.org/home/index.cfm?l=1\&id=4475 > [Consulta: 18 de octubre 2007].

2004. A New Chance for Haiti? Latin America/Caribbean Report 10, 18 November. [En línea] $<$ http:/ / www.crisisgroup.org/library/documents/latin_america/10\%20A $\% 20 \mathrm{New} \% 20$ Chance $\% 20$ for\%20Haiti.pdf>[Consulta: 11 de octubre 2007].

Keen, David. 1998. "The Economic Functions of Violence in Civil Wars" Adelphi Paper Series. Oxford: Oxford University Press for the International Institute for Strategic Studies.

Kreps, Sarah E. 2007. "The 1994 Haiti Intervention: A Unilateral Operation in Multilateral Clothes". The Journal of Strategic Studies 30 (3):449-74

Malloy, James. 1977. Authoritarianism and Corporativism in Latin America. Pittsburgh: Pittsburgh University Press.

Medicines Sans Frontiers.2004. Haiti: Assisting Victims of Violence and Flooding. [En línea] <http:/ / www.msf.org/msfinternational/invoke.cfm?objectid=B8EDB9FA-EF79-467C-B0FD3BB71F22E45B \&component=toolkit.article\&method=full_html>[Consulta: 19 de octubre 2007].

MINUSTAH. 2007. Hechos y Cifras. [En línea] <http://www.un.org/spanish/Depts/dpko/ minustah/>[Consulta: 19 de diciembre 2007].

Minear, Larry. 2005. The Humanitarian Enterprise: Dilemmas \& Discoveries. Bloomfield: Kumarian Press.

Nicolls, David. 1998. "The Duvallier Regime in Haiti”. En Sultanistic Regimes, editado por H.E. Chehabi y Juan Linz. Baltimore: Johns Hopkins University Press, 153-181..

Oficina del Alto Comisionado de Naciones Unidas para los Derechos Humanos. 2007. Situación sobre los derechos humanos en Haití. [En línea] < http:/ / daccessdds.un.org/doc/UNDOC/GEN/G07/105/75/ PDF/G0710575.pdf?OpenElement> [Consulta: 19 de octubre 2007].

Prensa Latina. 2007. Alertan sobre reanudación de secuestros en Haití. [En línea] < http:/ /news.caribseek. com/Haiti/article_58904.shtml [Consulta: 11 de octubre 2007].

Programa de Naciones Unidas para el Desarrollo. 2006. Human Development Report. [En línea] < http:/ / hdr.undp.org/en/statistics/> [Consulta: 11 de diciembre 2007].

Red de Seguridad y Defensa de América Latina. 2007- Atlas Comparativo de la Defensa en América Latina [En línea] < http:/ / www.resdal.org/haiti/main-haiti-crisis.html> [Consulta: 1 de diciembre 2007].

Reed, Lindsay. 2006. "Peace Despite the Peacekeepers in Haiti". NACLA Report on the America 39 (6): 31-37.

Stotzky, Irwin P. 1997. Silencing the Guns in Haiti: The Promise of Deliberative Democracy. Chicago: University of Chicago Press.

The Economist. 2007. "Building a Reluctant Nation". 382 (8515): 35.

The Economist. 2006. Making the Best of an election in a failed state. 378 (8465): 11-2.

The Economist. 2004. “The Blame Game". 373 (8398): 38.

The Economist Intelligence Unit. 2007. Haiti: Country Outlook. [En línea] <http://store.eiu.com/ product/50000205HT.html> [Consulta: 1 de enero 2008].

The Guardian. 2007. Haiti Emerges from a Nightmare, November 2.

The Miami Herald. 2007a. Haiti is not Out of the woods yet. September 10. 
Thompson, Andrew. 2007. "The Canadian Perspective for Research Agenda in Haiti". Paper delivered at the International Development Research Centre Workshop Reconstruction of Haiti: Research Capacity Building in Latin American Countries, Montreal Quebec 6 September.

UNICEF.2008. Secuestros de Niños en Haití. [En línea] <http:/ / www.un.org/spanish/News/fullstorynews. asp?NewsID=11048> [Consulta: 10 de enero 2008].

Juan Esteban Montes es Ph.D. (c) en Ciencias Políticas de la Universidad de Notre Dame. Abogado de la Pontificia Universidad Católica de Chile. Director del Programa en Chile de la Universidad de Notre Dame. Asesor del Subsecretario de Guerra, Ministerio de Defensa de Chile. Libros publicados: Subordinación Democrática de los Militares. Éxitos y Fracasos en Chile (1994) y ¿Y qué pasó con los militares? Chile, 1990-2002 (2003), ambos en coautoría con Gonzalo García.

(E-mail: jmontes@ssg.gov.cl)

Andreas Feldmann Pietsch es profesor auxiliar asociado del Instituto de Ciencia Política de la Pontificia Universidad Católica de Chile. Se especializa en Relaciones Internacionales. Sus temas de investigación incluyen el uso del terror como instrumento de violencia política, conflicto armado y migración forzada y derechos humanos. Desde el 2007 encabeza junto a Esteban Montes un proyecto financiado por el International Development Research Centre (IDRC) sobre difusión internacional de la temática de Haití en Chile.

(E-mail: afeldmann@uc.cl) 